\title{
PMS1 Homolog 2 Measurement
}

National Cancer Institute

\section{Source}

National Cancer Institute. PMS1 Homolog 2 Measurement. NCI Thesaurus. Code C139097.

The determination of the amount of PMS1 homolog 2 present in a sample. 